\title{
Efficacy of routine post-operative head computed tomography on cranial surgery patients outcome
}

Omar Qoqandi, MBBS, Abdulaziz O. Almubarak, MBBS, Mohammed Bafaquh, MBBS, FRCSC, Abdullah Alobaid, MBBS, FRCSC, Fahd Alsubaie, MBBS, FRCSC, Abeer Alaglan, MBBS, Dhuha A. Abukhamssin, MBBS, Mariam A. Algharib, MBBS, Abdulrazag I. Alsomali, MBBS, Mahmoud Alyamani, MBBS, FRCSC, Yasser orz, MBBS.

\footnotetext{
ABSTRACT

الأهداف : معرفة هل عمل هذه الأشعة له اي تأثير في القرارات

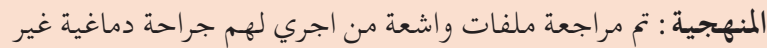

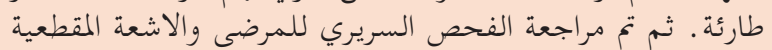

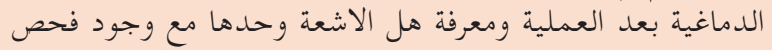

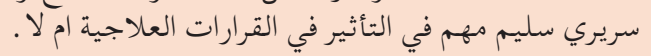

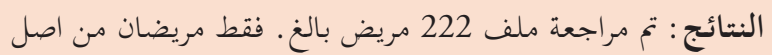

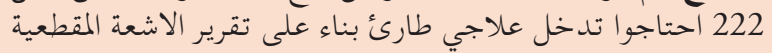

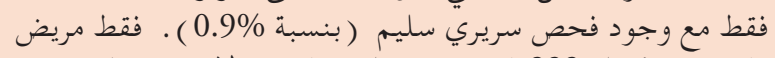

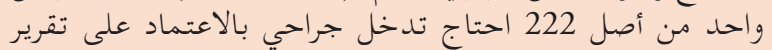
الاشعة فقط (بنسبة 0.4\%

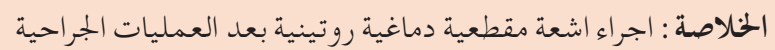

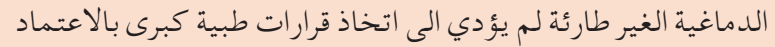

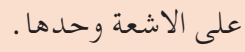

Objectives: To identify the role of routine postoperative head CT in changing postoperative management after elective craniotomies.

Methods: We conducted a retrospective study on adult patients who underwent cranial surgery. Exclusion criteria includes cranial CTs done postoperatively for urgent clinical indications, pediatric patients, CSF diversion procedures and sedated patients. Patients were placed into "positive" group if the physical assessment changed from the baseline in the form of clinical deterioration, and the "negative" group if the exam did not change. The data then were analyzed to identify which patients needed further medical or surgical management based on CT findings only with "negative" physical examination.

Results: Total of 222 were included in the study. 151 patients had negative physical examination. Only 8 out of 151 patients had positive CT findings. Two
}

patients out of $222(0.9 \%)$ had a negative physical exam and positive $\mathrm{CT}$ findings that required additional action that wouldn't be done urgently without routine postoperative brain CT. Only one patient out of 222 $(0.4 \%)$ who was re-operated urgently based CT findings only and negative physical examination.

Conclusion: Routine postoperative routine brain CT did not alter the course of medical management, even in the presence of significant radiological findings.

Neurosciences 2020; Vol. 25 (4): 281-286 doi: 10.17712/nsj.2020.4.20200035

From the National Neuroscience Institute (Qoqandi, Almubarak, Alaglan, Abukhamssin, Algharib, Alsomali, orz, Bafaquh, Alobaid, Alsubaie, Alyamani), King Fahed Medical City, from the College of Medicine (Abukhamssin, Algharib) Princess Nourah bint Abdulrahman University, Riyadh, from the Department of Neurosurgery (Almubarak), Prince Mohammed Medical City, Aljouf, from the College of Medicine (Alaglan), Qassim University, Qassim, Kingdom of Saudi Arabia.

Received 11th May 2020. Accepted 7th March 2020.

Address correspondence and reprint request to: Dr. Mohammed Bafaquh, National Neuroscience Institute, King Fahed Medical City, Riyadh, Kingdom of Saudi Arabia.E-mail : bafaquh@gmail.com ORCID ID: https://orcid.org/0000-0002-3829-4837

$\mathrm{N}$ eurosurgical patients have high early postoperative complications in comparison with other surgical domains. ${ }^{1}$ Postoperative brain CT is done routinely after cranial procedures to predict the possibility of early complications, including hemorrhage and significant brain edema. ${ }^{2}$ Other less urgent indications are the gross assessment of the extent of resection and ventricular catheter placement in cerebrospinal fluid diversion procedures. ${ }^{3}$ Imaging studies in our institute are done routinely for the postoperative cranial surgeries, even in the absence of clinical indication or postoperative complications; thus, raising suspicion about the role and efficacy of routine postoperative imaging. 
In this study, the aim is to determine the necessity of postoperative head CT scan in cranial surgeries to identify early complications, and its role in patient care, outcomes, and costs, with a review of the literature.

Methods. We conducted a retrospective observational study on adult patients who underwent cranial surgery in the inpatient department of adult neurosurgery at King Fahad Medical City in Riyadh. After Institutional Research Board approval, we reviewed patients records between January 2015 and December 2016. Any patient who underwent routine cranial CT scan postoperatively ( less than 24 hours from surgery) was included. Exclusion criteria involved cranial CT done postoperatively for clinical indications (neurological deterioration), pediatric patients less than 12 years of age, ventriculoperitoneal shunt surgeries, external ventricular drain placement surgeries, and sedated patients in whom clinical exam is not feasible.

Demographic data were collected that included age, gender, diagnosis, and type of surgery (e.g. aneurysm clip ligation). We reviewed the clinical status and physical examination done for each patient. Patients were placed into the "positive" group if the physical assessment changed from the baseline in the form of clinical deterioration, or new unexpected neurological deficits and the "negative" group if the patients had stable clinical status without new neurological findings. Physical examination data were retrieved from the patients' records. Brain CT scans from our (PACS) reviewed each patient for new significant findings (hemorrhage, brain edema, infarction, midline shift or herniation syndromes). Allocation to "positive" or "negative" CT findings was done subjectively based on the on-call radiologists through their written report or by the neurosurgeon. Any change in plans based on the physical examination or imaging was also retrieved. Management plan change based on first postoperative routine plain brain $\mathrm{CT}$ include emergency surgery, escalation in service from high dependency unit (HDU) to neuro intensive care unit, admiration of new medication (mannitol), or further imaging. Data were analyzed using IBM SPSS version 25 statistical software.

Results. We reviewed a total of 222 patients. Demographic data and the type of procedures are

Disclosure. Authors have no conflict of interests, and the work was not supported or funded by any drug company. summarized in Table 1 . The procedures conducted are 195 craniotomies, 12 craniectomies, and 15 burr holes for various indications. Out of the 222 patients, 151 patients had negative physical examination, defined as no change in the neurological status from the preoperative baseline or expected deficits postoperatively with no clinical deterioration in the early postoperative period. Only 8 out of 151 patients had positive CT findings (Table 2). These findings include significant hemorrhage at the surgical site, territorial infarctions, edema with mass effect, partial effacement of basal cisterns, or sizable residual tumor causing a significant mass effect. Modifications of clinical decisions occurred in 2 out of 8 patients based on CT findings only. In one patient, the finding included the presence of subdural hemorrhage causing a mass effect which necessitated another follow-up brain CT in less than $24 \mathrm{~h}$ and eventually managed conservatively. The other patient had a residual of his hemorrhagic tumor and was taken back to the theater for resection on the same day.

Seventy-one patients had a positive physical examination in the form of new neurological deficits, altered level consciousness, clinical instability, or deterioration. Out of these patients, only 24 had positive CT findings (Table 2). Change in management based on CT brain was done for four patients only, although all the four have abnormal physical examination. One patient underwent tumor resection, and was given heparin infusion the next day due to a metallic cardiac valve; his CT showed diffuse subarachnoid hemorrhage, he had a fluctuating level of consciousness on examination. The heparin infusion was stopped, and additional follow-up brain imaging was requested. The second patient underwent medial sphenoid wing meningioma resection; postoperative CT brain showed hypodensity in middle cerebral artery distribution, examination

Table 1 - Characteristics of the patients included in the study: age, gender, primary disease category and type of procedure.

\begin{tabular}{lc}
\hline Characteristics & $\mathbf{n}(\%)$ \\
\hline Age & Average 46 years $(18-77)$ \\
$\quad$ Gender & $122(55)$ \\
Female & $100(45 \%)$ \\
Male & \\
Disease Category & $136(61.2 \%)$ \\
Neoplasm & $77(34.6 \%)$ \\
Vascular & $4(1.8 \%)$ \\
Infectious & $5(2.2 \%)$ \\
Functional/Epilepsy & \\
Type of Procedure & $195(87.8 \%)$ \\
craniotomy & $12(5.4 \%)$ \\
craniectomy & $15(6.7 \%)$ \\
Burr hole & \\
\hline &
\end{tabular}


Table 2 - Showing positive and negative findings in neurological examination in less than 24 from surgery and corresponding's routine postoperative CT brain findings.

\begin{tabular}{lccc}
\hline CT Brain Findings & \multicolumn{2}{c}{ Neurological Examination } \\
Positive & Negative & Total \\
$\mathbf{n}(\%)$ & \\
\hline Positive* & $24(10.8)$ & $8(3.6)$ & $32(14.4)$ \\
Negative & $47(21.2)$ & $143(64.4)$ & $190(85.5)$ \\
Total & $71(31.9)$ & $151(68)$ & $222(100)$ \\
\hline *Presence of abnormal worrisome finding (e.g. mass effect, significant hematoma, \\
massive infarction). ${ }^{* *}$ Presence of abnormal unexpected new clinical deterioration. \\
\hline
\end{tabular}

revealed hemiparesis. A diagnosis of vasospasm was made with an additional angiogram; he was started on vasospasm protocol and kept in neuro ICU. The third patient underwent anterior communicating artery aneurysm clip ligation; postoperative brain CT showed diffuse brain swelling and partial effacement of basal cisterns; he was kept in neuro intensive care unit for close observation. The last patient underwent left occipital craniotomy and evacuation of intracerebral hematoma; CT showed sizable epidural hematoma at the surgical bed. The patient was retaken urgently to the operative theater for evacuation of the hematoma.

In total, only 6 patients $(2.7 \%)$ required urgent action postoperatively on day one. However, only 2 patients out of $222(0.9 \%)$ had a negative physical exam and positive CT findings that required additional action that would not be done urgently without routine postoperative brain CT. Only one patient out of $222(0.4 \%)$ who was re-operated urgently based CT findings only and negative physical examination. The other 4 patients also had a significant abnormal physical examination that would usually require urgent brain imaging.

Illustrative case. A 44-year-old male, known to have ischemic heart disease, presented to emergency department with confusion, headaches, blurring of vision, forgetfulness, seizures, right lower limb numbness and weakness for two months. He had GCS of $14 / 15$, with normal power, sensory, and cranial nerves. MRI brain showed large intra-axial mass involving the left frontal lobe and $8 \mathrm{~mm}$ midline shift. The patient underwent left frontal craniotomy and tumor resection He was extubated and shifted to ICU. His postoperative clinical examination did not differ from the baseline. Postoperative routine brain CT was done the next day morning and showed mild subdural hematoma and a large hematoma in the surgical bed with left frontal edema. A compression was caused by the hematoma on the left frontal horn of the lateral ventricle with a rightwards midline shift of $10 \mathrm{~mm}$. The patient was kept under observation; no clinical deterioration or change in baseline occurred. Repeated brain CT in three days showed the same hematoma with intermediate resolution. Pathological examination revealed a high-grade glioma.

Discussion. Routine postoperative brain CT after a cranial procedure is common practice in neurosurgery that relies on physician preference. In this article, we found that the use of routine postoperative brain CT

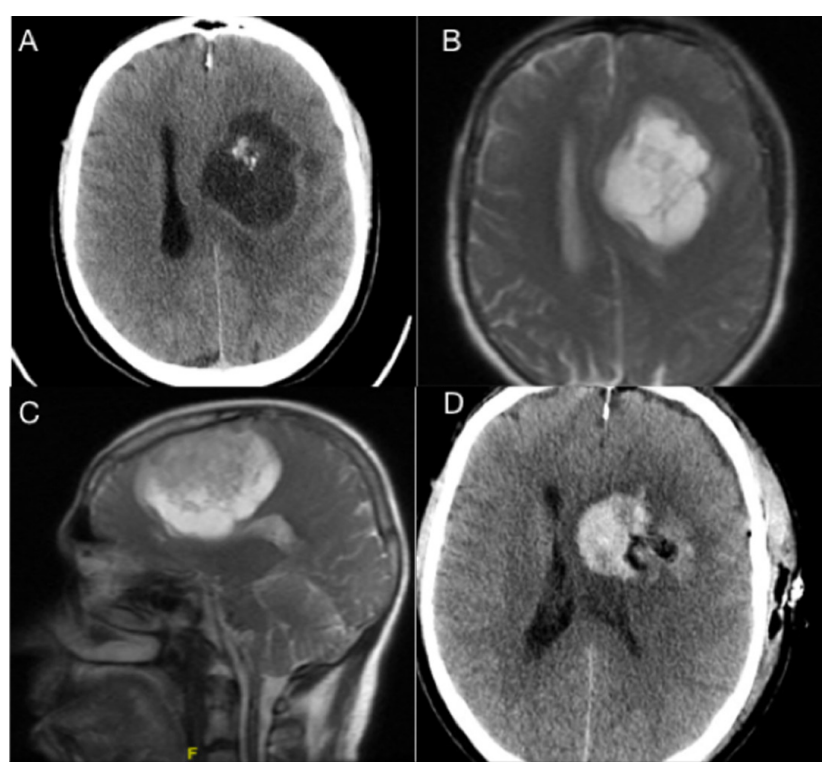

Figure 1- Perioperative imaging of elective craniotomy and removal of left frontal mass. A) peroperative plain CT brain showed left frontoparietal subcortical hypodense lesion with central hyperdensity causing mass effect and compression on adjacent structures (arrow). B,C) Axial and sagittal peroperative MRI showing the same lesion, hyperintense on $\mathrm{T} 2$ weighted images (arrows). D) routine postoperative CT brain showing surgical site hematoma with compression on ipsilateral body of left lateral ventricle (arrow), causing midline shift. 
does not alter the course of management, regardless of the presence or absence of significant findings. We estimated that the percentage of escalation of management and urgent re-operation based on routine postoperative brain CT and negative physical examination was only $0.9 \%$ and $0.4 \%$, respectively. Most of the patients with significant findings on postoperative brain imaging also had abnormal physical examination. These findings do not support the use of routine postoperative brain CT in cranial procedures. The presence of significant findings on early routine postoperative brain CT was $14.4 \%$ in our study; however, only $0.9 \%$ required intervention or or change in the management based on CT findings ony. Presence of positive findings upon brain CT in stable patients does not justify surgical intervention. In fact, it may result in further unnecessary workup, imaging, or increase length of hospital stay.

Studies in the literature addressed the utility of the routine postoperative CT in neurological surgeries, proving the importance of its role in serving the patient, with evidence-based practices. The above findings are consistent with a recent study on 666 patients. Their re-operation rate was $2.7 \% \quad(n=18)$, however, all re-operated patients had new neurological deficits before CT. ${ }^{4}$ Schär et $\mathrm{al}^{2}$ examined the utility of brain CT on 469 patients and concluded that routine imaging is not necessarily on patients who are conscious. Their emergency re-operation rate within 48 hours based on CT findings was $1.6 \%$, close to our figure. Furthermore, a study conducted on all neurosurgical patients to examine the importance of postoperative brain CT, 464 craniotomies/craniectomies out of 892 routine postoperative CT procedures did not necessitate any further surgical intervention. ${ }^{5}$ However, none of these studies examined the changes in the none-surgical component of management (close neurosurgical observation, escalation of service, changing medication), but all of them supported the conclusion that re-operation was not necessary based on CT findings only in conscious, neurologically-stable patients without clinical deterioration.

In neurosurgical patients who underwent procedures other than craniotomies, early postoperative brain CT is not necessary. Nadimi et $\mathrm{al}^{6}$ reported that in anterior endoscopic skull base surgeries, the re-operation rate based on CT findings was $2 \%$ in the 143 patients who underwent the procedure for various indications; all of them had expanding pneumocephalus. Their study also supports our findings that early brain CT is not necessary; however, in the patients urgently re-operated on, they used early postoperative brain CT as a baseline to assess the extent of pneumocephalus expansion.
This was confirmed by another study on endoscopic ventral skull base surgeries. Any abnormality observed upon brain CT is usually accompanied by clinical deterioration; therefore, routine postoperative imaging is not necessary unless indicated. ${ }^{7}$ Patients with chronic subdural hematoma underwent burr-hole evacuation and drain insertion, and early cranial imaging did not show reduced recurrence or improved outcome; in fact, it increased the length of hospital stay. ${ }^{8}$ In a previous study conducted at Rush University, 433 procedures out of 892 were burr holes, angioplasties, or transsphenoidal surgeries, and early brain CT did not show any improvement in re-operation rates based on CT findings only. ${ }^{5}$

We consider that there are certain cases where routine postoperative brain CT is justified. In a retrospective study of craniosynostosis reconstructive surgeries, the imaging findings were not found to be clinically significant in patients without shunt placement after the reconstruction; however, in patients with implanted shunts, doing brain CT is justified due to a high incidence (29\%) of shunt malfunction. ${ }^{9}$ Furthermore, the point of medico-legal reasons to do routine postoperative CT scans to rule out hematoma was raised in other studies, ${ }^{10,11}$ despite their low risk $(0.69 \%) .{ }^{11}$ In our study, only one patient was taken back to operating theater urgently due to CT findings only. Although the back-to-theater rate is low in our study $(0.4 \%)$, some argue that this figure is still reasonable to conduct a routine post operative CT brain in neurosurgical patients and it's cost-effective. In comatose or sedated patients, routine postoperative brain CT is justified as the ICP monitor alone is not reliable in detecting focal neurological injury or compression, ${ }^{12}$ which can be detected in the neurological exam if the patient is conscious. ${ }^{13}$ In comatose patients who are in postoperative period, the risk of CT changes is six times higher than in the non-comatose patients, and they are at twice the risk to go for the second intervention than the non-comatose patients. ${ }^{14}$ The literature extensively supports the use of routine post-craniotomy brain CT in the sedated or comatose patients when the clinical neurological exam is not possible. Furthermore, one of the arguments with the use routine postoperative CT brain is to obtain it as a baseline study for future comparison in case of deterioration or late complications or to initiate early pharmacological venous thromboembolic prophylaxis. Certain centers obtain early brain CT as part of their early discharge plan pathway to exclude any postoperative hematomas.

Positive clinical findings in patients with negative 
CT brain can be explained by multiple reasons, this includes CT-occult early vascular insults that can be better detected with MRI, electrolytes imbalance (most commonly being hyponatremia), ${ }^{15}$ anesthetic/ analgesic agents or anti-epileptic drugs, ${ }^{16}$ status of high ICP without changes in the ventricular system ${ }^{17}$ and extracranial related conditions (e.g. peripheral nerve injury due to positioning or spine related problems). ${ }^{18}$ Regarding ICU stay, patients who have findings in the postoperative CT scan are divided into 2 categories; first, radiological findings associated with neurological deficits. These represent a small number of patients that require an extension of ICU stay or step-down unit till resolution of the problem, however, some of these patients were transferred to regular ward beds, depending on the clinical and radiological assessment. The second category is patients with non-neurological deficits. These patients were transferred to a ward bed in most cases (70\% from this category) and only a few have a longer ICU length of stay.

Other factors should be considered when doing routine postoperative brain CT is radiation exposure. A routine head CT is $2.1 \mathrm{mSv}$, which is equivalent to 30 chest $\mathrm{x}$-rays. ${ }^{19}$ In the pediatric group, $2-3$ head CTs can triple the risk of brain tumors. ${ }^{20}$ In neurosurgical practice, brain CT is the first and the most commonly used imaging modality. Neurosurgical patients are commonly scanned for neurosurgical conditions, including postoperative routine imaging, ER or inpatients clinical suspicion of deterioration, or even routine follow-ups in clinics. This substantially increases the risk of radiation exposure.

In terms of cost-effectiveness in obtaining postoperative head CT in neurosurgical patients, aneurysm patients with focal neurological deficit require 11 head CT scans at the cost of $\$ 3212$ to obtain a CT that would change the clinical management, but in neurologically intact patients, it needs 99 brain scans at the cost of $\$ 28,908$ to get a single CT that would alter the medical management. ${ }^{12}$ Performing head CT in neurologically intact patients suspected to have ongoing intracranial insult preceding neurological deterioration is not an uncommon scenario. Ordering head CT brain should be guided and individualized based on clinical examination and continuous monitoring in ICU settings to utilize hospital resources and avoid devastating postoperative complications.

There are multiple limitations in our study. Being a retrospective study with a moderate sample size. The significant findings on the brain CT were based on subjective interpretation of the viewer without objective parameters. The rate of significant postoperative CT findings in this study $(14.4 \%)$ is high, and could explained by the subjectivity of images interpretations and relaying on the radiologist report. Another important limitation is the inability to follow and retrieve all post-operative medical management/ decisions and identify which of them was done based on the postoperative imaging, only major and obvious medical decision were included in this study. Further controlled prospective studies are needed to draw firm conclusion on this manner.

In conclusion, the use of early postoperative routine brain CT $(<24 \mathrm{~h})$ in elective cranial surgery in conscious patients, without any changes in the neurological examination, did not alter the course of medical management, even in the presence of significant radiological findings.

\section{References}

1. Manninen PH, Raman SK, Boyle K, el-Beheiry H. Early postoperative complications following neurosurgical procedures. Can J Anaesth 1999; 46: 7-14.

2. Schar RT, Fiechter M, Z'Graggen WJ, Soll N, Krejci V, Wiest R, et al. No Routine Postoperative Head CT following Elective Craniotomy--A Paradigm Shift? PLoS One 2016; 11: e0153499.

3. Khaldi A, Prabhu VC, Anderson DE, Origitano TC. The clinical significance and optimal timing of postoperative computed tomography following cranial surgery. J Neurosurg 2010; 113: 1021-1025.

4. Freyschlag CF, Gruber R, Bauer M, Grams AE, Thomé C. Routine Postoperative Computed Tomography Is Not Helpful After Elective Craniotomy. World neurosurgery 2019; 122: e1426-e1431.

5. Fontes RB, Smith AP, Munoz LF, Byrne RW, Traynelis VC. Relevance of early head CT scans following neurosurgical procedures: an analysis of 892 intracranial procedures at Rush University Medical Center. J Neurosurg 2014; 121: 307-312.

6. Nadimi S, Caballero N, Carpenter P, Sowa L, Cunningham $\mathrm{R}$, Welch KC. Immediate postoperative imaging after uncomplicated endoscopic approach to the anterior skull base: is it necessary? Int Forum Allergy Rhinol 2014; 4: 1024-1029.

7. Diaz L, Mady LJ, Mendelson ZS, Liu JK, Eloy JA. Endoscopic ventral skull base surgery: Is early postoperative imaging warranted for detecting complications? The Laryngoscope 2015; 125: 1072-1076.

8. Brokinkel B, Ewelt C, Holling M, Hesselmann V, Heindel WL, Stummer W, et al. Routine postoperative CT-scans after burr hole trepanation for chronic subdural hematoma - better before or after drainage removal? Turk Neurosurg 2013; 23: 458-463.

9. Binning M, Ragel B, Brockmeyer DL, Walker ML, Kestle JR. Evaluation of the necessity of postoperative imaging after craniosynostosis surgery. J Neurosurg 2007; 107: 43-45.

10. Wen L, Yang XF, Jiang H, Wang H, Zhan RY. Routine early CT scanning after craniotomy: is it effective for the early detection of postoperative intracranial hematoma? Acta Neurochir (Wien) 2016; 158: 1447-1452. 
11. Hussain SA, Selway R, Harding C, Polkey CE. The urgent postoperative CT scan: a critical appraisal of its impact. $\mathrm{Br} J$ Neurosurg 2001; 15: 116-118.

12. Zygourakis CC, Winkler E, Pitts L, Hannegan L, Franc B, Lawton MT. Clinical utility and cost analysis of routine postoperative head CT in elective aneurysm clippings. $J$ Neurosurg 2017; 126: 558-563.

13. Zapolska G, Komarowska M, Turek G, Hermanowicz A, Matuszczak E, Zielinska-Turek J, et al. "Talk and deteriorate" syndrome and the role of an immediate follow-up computed tomography of the head in children. Pediatria Polska 2016; 91: 155-160.

14. Alkhalili K, Zenonos G, Tataryn Z, Amankulor N, Engh J. The Utility of Early Postoperative Head Computed Tomography in Brain Tumor Surgery: A Retrospective Analysis of 755 Cases. World neurosurgery 2018; 111: e206-e212.

15. Hannon MJ, Finucane FM, Sherlock M, Agha A, Thompson CJ. Disorders of Water Homeostasis in Neurosurgical Patients. J Clin Endocrinol Metab 2012; 97:1423-1433.
16. Wu AS, Trinh VT, Suki D, Graham S, Forman A, Weinberg JS, et al. A prospective randomized trial of perioperative seizure prophylaxis in patients with intraparenchymal brain tumors. $J$ Neurosurg 2013; 118: 873-83.

17. Børgesen SE, Gjerris F. Relationships between intracranial pressure, ventricular size, and resistance to CSF outflow. $J$ Neurosurg 1987; 67: 535.

18. Hewson DW, Bedforth NM, Hardman JG. Peripheral nerve injury arising in anaesthesia practice. Anaesthesia 2018; 73: 51-60.

19. Smith-Bindman R, Lipson J, Marcus R, Kim KP, Mahesh M, Gould R, et al. Radiation Dose Associated with Common Computed Tomography Examinations and the Associated Lifetime Attributable Risk of Cancer. Arch Intern Med 2009; 169: 2078-2086.

20. Pearce MS, Salotti JA, Little MP, McHugh K, Lee C, Kim KP, et al. Radiation exposure from CT scans in childhood and subsequent risk of leukaemia and brain tumours: a retrospective cohort study. The Lancet 2012; 380: 499-450.

\section{Illustrations, Figures, Photographs}

All figures or photographs should be submitted in a high resolution (minimum $300 \mathrm{DPI}$ ) electronic version saved in jpeg or tiff format. Original hard copies of all figures may be requested when necessary. Photographs will be accepted at the discretion of the Editorial Board. All lettering, arrows, or other artwork must be done by an artist or draftsman. If arrows are used please ensure they appear in a different color to the background color, preferably black with a white border, or white with a black border. If arrows distinguish different items on the figure then different arrow styles should be used ie. long, short, wide, narrow. Written informed consent for publication must accompany any photograph in which the subject can be identified. Written copyright permission, from the publishers, must accompany any illustration that has been previously published. 